\title{
Leapfrog tracking for the storage ring pEDM project
}

\author{
Alfredo U. Luccio \\ ${ }^{1}$ (guest) Brookhaven National Laboratory, Upton, NY, USA \\ e-mail: luccio@bnl.gov
}

\begin{abstract}
We are planning an experiment to measure the Electric Dipole Moment of the proton with the sensitivity of $10^{-29} \mathrm{e} \cdot \mathrm{cm}$ using an electrostatic storage ring. In this paper we describe a tracking simulation for the project based on the Leapfrog algorithm.
\end{abstract}

\section{Introduction}

We plan a storage ring based experiment to measure the Electric Dipole Moment (EDM) of the proton with an accuracy of $10^{-29} \mathrm{e}-\mathrm{cm}$. In the pEDM we will use polarized protons at the magic momentum of $0.7 \mathrm{Gev} / \mathrm{c}$. The EDM will be measured by spin polarimetry.

The rings will be electrostatic (no magnets) and in such a lattice the spin of a proton of magic momentum will remain "frozen" in its direction at injection (longitudinal) and the EDM will be measured as proportional to a small vertical component of the spin that will gradually appear, see Sec.5, that will be measured.

Spin dynamics is governed by the covariant Thomas-Bargman-Michel-Telegdi (T-BMT) equation

$$
\frac{d \mathbf{s}}{d t}=-\frac{q}{m \gamma} \mathbf{f} \times \mathbf{s}
$$

where $\mathbf{s}$ is the real 3-dimensional spin vector of a 1/2-spin particle, and $\mathbf{f}$ is a function of the position and the momentum of the particle and of the (laboratory) electric and magnetic field encountered by the particle along its trajectory. Spin is a passenger on the orbit, that will be treated in this paper. In a pure electrostatic ring $\mathbf{f}$ reduces to

$$
\mathbf{f}=\left(a \gamma-\frac{\gamma}{\gamma^{2}-1}\right) \frac{\mathbf{E} \times \mathbf{v}}{c^{2}},
$$

with $a$ the spin anomaly. At the magic momentum $p c=m c^{2} / \sqrt{a}$ it is exactly $\mathbf{f}=\mathbf{0}$, and the spin will not precess at all if the EDM is zero.

\section{Orbit/Spin Tracking in an electric ring}

Simulation of an electrostatic storage ring for the pEDM is important and should be done by more than one method to compare and benchmark. Tracking should be symplectic for stability in the long range

\footnotetext{
${ }^{1}$ We acknowledge the support of the U.S. Department of Energy
} 
and fast, because ring turns will be many. Tracking a pure electric lattice is different than tracking a lattice based on magnetic bends, which is the object of much of the standard Accelerator Physics, for two main reasons: (1) the kinetic energy of a particle changes in traversing an electric field (its total energy does not), (2) the bending field within a bend changes with the radial position of the particle, while in a magnetic bend the field can be considered approximately uniform.

There are orbit tracking codes using:

(1) Integration of differential equations for orbit (Lorentz) and spin (Thomas-BMT) with Runge-Kutta type routines,

(2) Map description of single machine elements or of the whole lattice,

(3) Symplectic integration for propagation by discrete kicks.

Kick propagation is the method we will describe In the following. It is a Leapfrog kick code that performs the symplectic integration of the Lorentz equation of motion, with only the electric field $\mathbf{E}$ (the over-braced part vanishes in an electric ring, and will be only briefly used for benchmarking.)

$$
\frac{d \mathbf{p}}{d t}=e \mathbf{E}+\overbrace{e \mathbf{B} \times \mathbf{v}} \text { with }: \mathbf{E}=-\nabla \boldsymbol{\Phi}, \overbrace{\mathbf{B}=\nabla \times \mathbf{A}}
$$

The potentials $\Phi$ and $\mathbf{A}$, needed for the Hamiltonian, in static fields, will obey the equations

$$
\nabla^{2} \Phi \equiv \frac{\partial^{2} \Phi}{\partial x^{2}}+\frac{\partial^{2} \Phi}{\partial y^{2}}+\frac{\partial^{2} \Phi}{\partial z^{2}}=0, \quad \nabla \cdot \mathbf{A}=0, \quad \nabla \times \mathbf{A}=0
$$

Formulation for $\Phi(x, y, z)$ and $\mathbf{A}(x, y, z)$ in the tracking code are found by power expansion.

The Leapfrog kick method to integrate differential equations of motion was invented by the astronomer Delambre in 1792 [1], then revived by Verlet in 1960 [2], so the method is also known as 'Verlet' kicks. Leapfrog integration was introduced for accelerators in a seminal 1983 paper by Ronald Ruth[3], and also forms the basis of a general symplectic integration algorithm by the same Author. Similar algorithms are also used in the tracking code TEAPOT by L.Schachinger and R.Talman [4].

Leapfrog integration interleaves drifts, where only the space coordinates are advanced, with symplectic kick bends where the momentum components are advanced. Kicks are rather coarse and this makes the method fast. Leapfrog in its simplest form is accurate to 2.nd order in time step. Higher order has also been studied.

Other integration algorithms, like Runge-Kutta with tiny steps, are accurate to 4.th order in time. However they were written with mathematical accuracy in mind, while the 2.nd order Leapfrog is exactly symplectic, i.e. was written with physical accuracy in mind. Making also Runge-Kutta symplectic has been discussed [5], and reviewed i.a. in 'Numerical Recipes' [6] but RK makes a computer code slower to run, which defies our goal of short computer time for tracking the many turns of a pEDM ring.

Leapfrog tracking conserves the value of the Hamiltonian, that is being continuously recalculated during runs.

$$
\mathcal{H}=\sqrt{(\wp-e A)^{2}+\left(m c^{2}\right)^{2}}+e \phi
$$

Much of the standard accelerator tracking is done in the system of Frénet-Serret "accelerator" coordinates. Here, we use Cartesian "laboratory" coordinates $(x, z, y)$, with $\hat{y}$ vertical axis, and time as the independent variable, see Fig.1. Electric field components and potentials are calculated by a power expansion out of the "horizontal" $x, z$ plane of the ring. 
A ménagerie of quantities for the game is

\begin{tabular}{|ll|}
\hline$r_{o}[m]=$ radius of curvature, & $a=$ magnetic anomaly \\
$U_{o}=m c^{2}[\mathrm{GeV}]$ mass energy, & $\wp_{o} \equiv p c[\mathrm{GeV}]=U_{o} / \sqrt{a}$ magic momentum \\
$\wp=\wp_{o}(1+d p / p)$, & $U_{T}[\mathrm{GeV}]=\sqrt{\left(\wp^{2}+U_{o}^{2}\right.}$ total energy, $\gamma=U_{T} / U_{o}$ \\
$B \rho[V \cdot s / m]=10^{9} \wp / c$ rigidity, & $e E[e V / m]=\left(\wp / r_{0}\right) \beta c$ electric bend field \\
\hline
\end{tabular}

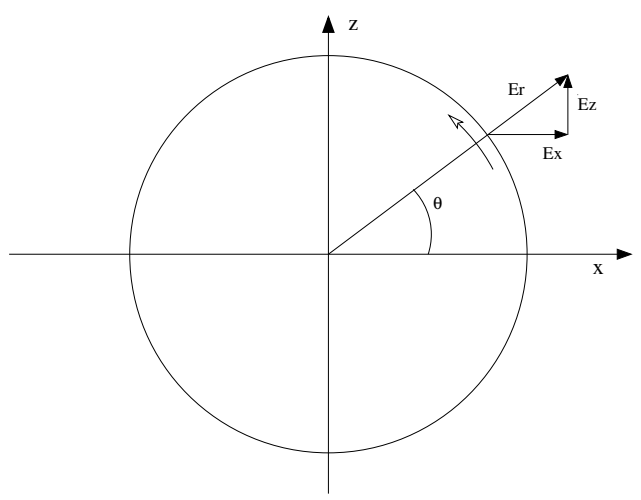

Figure 1. Reference Cartesian coordinates. $\hat{y}$ is out of the plane. The circular ring lattice shown is designed by tracking a "reference particle" i.e at the nominal, magic, energy injected tangentially.

\subsection{Orbit Leapfrog formalism basics}

Momentum kick are done by integration of the Lorentz equation Eq.(3), for an electric or magnetic bend, respectively. We will work on 3 examples (1) circular ring, (2) 8-super-period structure with 8 bends, 8 drifts and 8 electrostatic quadrupoles, (3) simple magnetic structure (helix). Figure 2 describes a basic LeapFrog cell in a bend. Start with a particle in the horizontal plane.

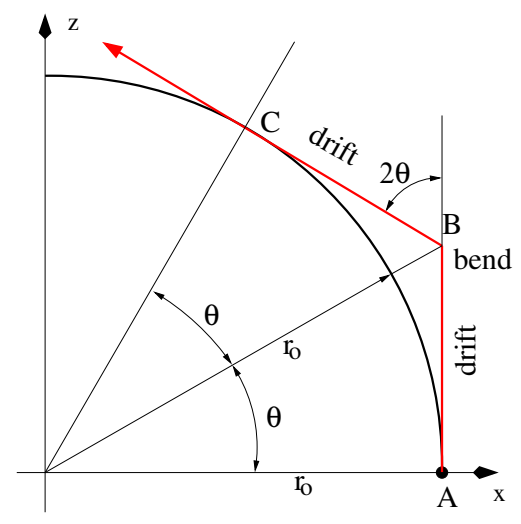

Figure 2. Leapfrog bend cell: drift, kick-bend, drift 
Basic leapfrog cell is a sequence [drift + momentum kick + drift], see Figure 2.

The reference particle, around which the whole beam dances, is the magic particle whose spin would remain frozen in position during the propagation. See what happens in a bend of the lattice to a reference particle in the horizontal plane. Start in $\mathbf{A}$ with initial coordinates

$$
\text { (A) } x=r_{o}, z=0, \wp_{x}=0, \wp_{z}=\wp .
$$

Eq's for the drift, with a time step $d t$ for the drift $\mathbf{A} \rightarrow \mathbf{B}$ :

$$
\left\{\begin{array}{l}
\frac{d x}{d t}=\frac{\wp_{x}}{U_{o} \gamma} c \rightarrow x:=x+\wp_{x} /\left(U_{o} \gamma\right) c d t \\
\frac{d z}{d t}=\frac{\wp_{z}}{U_{o} \gamma} c \rightarrow z:=z+\wp_{z} /\left(U_{o} \gamma\right) c d t
\end{array}\right.
$$

using the identity $\wp=U_{o} \beta \gamma$, we obtain at the kick bend $\mathbf{B}$ the new position

$$
\text { (B) } x=r_{o}, z=\beta c d t, \wp_{x}=0, \wp_{z}=\wp .
$$

In $\mathbf{B}$ a kick is imparted to the momentum $\wp$, using the Lorentz Equation, with a time step $\delta t$, different from the $d t$ of the drift.

$$
\wp_{x}:=\wp_{x}-e E_{x} c \delta t, \wp_{z}:=\wp_{z}-e E_{z} c \delta t .
$$

For cylindrical electrodes bend the field seen by the reference particle $E$ is purely radial, with components

$$
e E_{x}=-e E\left(r_{o} / r\right) \cos \theta, \quad e E_{z}=e E\left(r_{o} / r\right) \sin \theta .
$$

Find the relation between $d t$ and $\delta t$ for leapfrog that satisfies two conditions:

(1) Through the bend the value of the total momentum $\wp$ must be conserved

(2) The trajectory in $\mathbf{C}$ should return tangent to the circle, as in the figure. Namely:

$$
\arccos \left[(\mathbf{p}(A) \cdot \mathbf{p}(C)) / p^{2}\right]=2 \theta
$$

If both conditions hold, the basic trajectory will be a polygon circumscribed to the circle. Other particles in the beam will wiggle around it in betatron oscillations.

For condition (1): moment conservation, combining the preceding equations

$$
\wp_{x}=-\wp / r \cos \theta \beta c \delta t, \wp_{z}=\wp(1-(1 / r) \sin \theta \beta c \delta t)
$$

then after kick $(\mathbf{C})$ :

$$
\wp_{x}^{2}+\wp_{z}^{2}=(p c)^{2}\left[1+((\beta c / r) \delta t)^{2}-(2 / r) \sin \theta \beta c \delta t\right] .
$$

Since: $\cos \theta=z / r, \sin \theta=x / r$, taking the value of $x$ from Eq.(6), the term in [ ] in Eq.(11) above reduces to 1 when (the important LeapFrog rule)

$$
\delta t=2 d t
$$

For condition (2): the new new trajectory angle can be calculated from the scalar product of the momentum before and after the kick

- (A) before kick: $\wp_{x}=0, \wp_{z}=\wp$

- (C) after kick: $\wp_{x}=-(\wp / r) \cos \theta \beta c \delta t, \wp_{z}=\wp\left(1-2 \sin ^{2} \theta\right)$ 


$$
\text { angle }=\arccos \frac{\wp(A) \cdot \wp(B)}{(p c)^{2}}=\arccos \left(1-2 \sin ^{2} \theta\right)=2 \theta
$$

under the condition of Eq,(12), as desired.

Note that, in the general case of a particle injected at some angles or not at the reference energy, the leapfrog conditions (1) and (2) for momentum and angle are not fully satisfied in a bend because, due to transverse betatron oscillations, the particle sees a tangential component of the electric field that modulates the energy.

\subsection{Strategy to track a general lattice with Leapfrog}

An electrostatic lattice behaves differently than a classical magnetic lattice, as said in Sec.2. In an e.element the kinetic energy of a particle is modulated, while in a m.element it is not, since in the Lorentz equation of motion (3) only the scalar driving term appears. In a m.lattice it is the vector term to act, where the force is perpendicular to the velocity. However, the differences are not very great.

Much of the classic formalism of accelerator physics still holds and we can still preliminary design an e.lattice as an m.lattice to start the game, using the CERN MAD code [7]. We will design a stable m.storage ring to be converted afterwards to an e.ring, We need to address i.a. the modulation of energy and focusing in an electrostatic bend. To produce a gradient focusing m.bend, like in the Brookhaven's AGS, some focusing quadrupoles will be added in MAD. Similarly, we will deal with electrostatic multipoles, used for focusing and chromaticity correction.

To simulate in MAD the horizontal focusing of an e.bend (for cylindrical e.bends) we will introduce in the madx input. for each $\mathbf{B}$ bend location, an half quadrupole as an element of MAD type MATRIX , that only contains the upper-left 4 matrix elements as non-zero.

EMAT: MATRIX L $=\ell$ (see MAD User's manual.)

$$
\begin{gathered}
\left(\begin{array}{cc}
c_{x} & s_{x} \\
-k_{x}^{2} s_{x} & c_{x}
\end{array}\right) \\
c_{x}=\cos (k \ell), s_{x}=\sin (k \ell), \ell=c \delta t, k_{x}=e E /\left(\wp r_{o}\right),
\end{gathered}
$$

consistent with Eq.(8). Remember that MAD is written in "accelerator" coordinates, with " $\mathrm{x} " \equiv$ "radial". In the case of more complicated bend electrodes (spherical, toroidal) that also produce vertical focusing, this quadrupole will be written as a full $(4 \times 4)$ or even $(6 \times 6)$ matrix.

\section{Reference trajectory in a general lattice}

In the stable lattice, on the horizontal plane, we will produce a reference trajectory by Leapfrog tracking along a polygonal pattern tangent to a structure made of straights (drifts) and circular arcs (bends), The leapfrog polygonal orbit is slightly longer than the reference orbit. The more kicks we put in a bend the lesser this difference is.

The example of a structure with 8 bends and 8 drifts of circa $270 \mathrm{~m}$ of total length, using 32 kicks in each bend of $36 \mathrm{~m}$ of radius, is shown in Figure $3 \mathrm{~A}$. The difference in effective radius between the geometrical base line and the polygon is about $1 \mathrm{~mm}$. A complication in the case of a general lattice with bends and drifts is that the instantaneous center of curvature needed for electric field calculation in the bends should be re-evaluated every time, as hown in Figure 3B.

The Leapfrog formalism extends to 3 dimensions and applies unchanged to particles that don't have a magic energy or are injected in the lattice on a finite transverse emittance. Eqs.(6) and .(7) in 
EPJ Web of Conferences
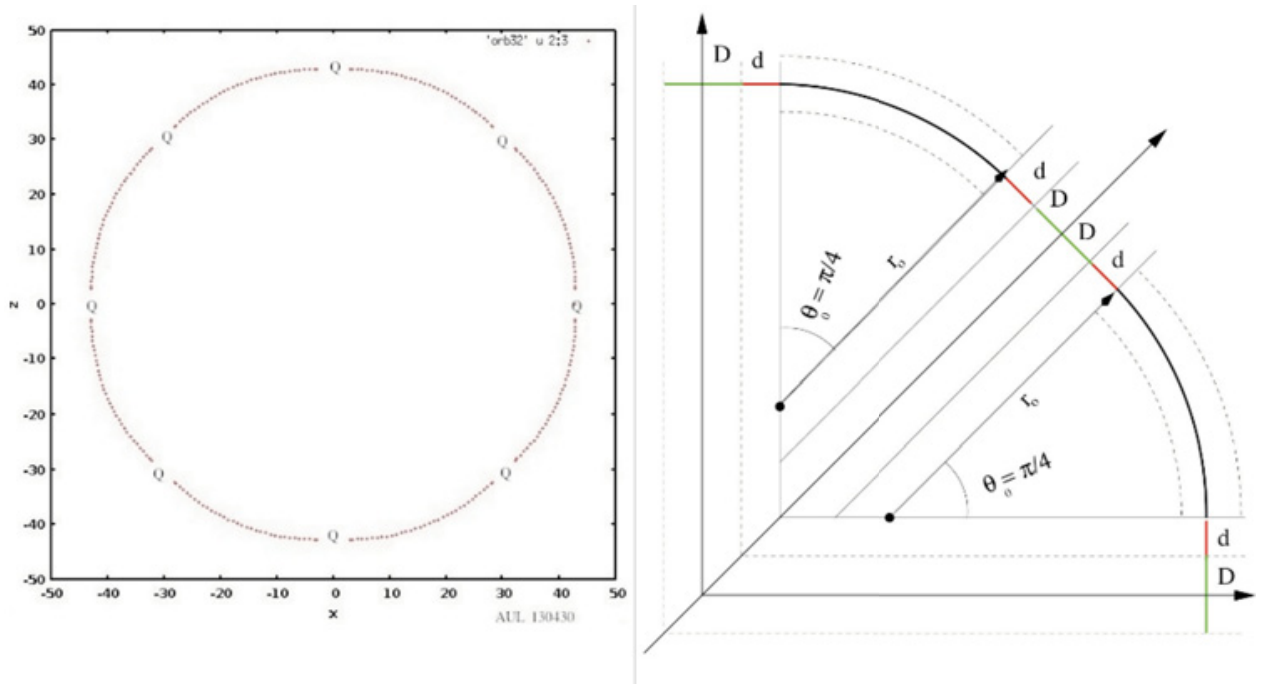
A. 32 kicks per bend, bend length $=28.276 \mathrm{~m}$, drift length $=2 \times 2.83 \mathrm{~m}$, intra bend drift length $=0.44 \mathrm{~m}$, nominal curvature radius $=36 \mathrm{~m}$,
$E_{x y}=-1.164745510^{7} \mathrm{~V} / \mathrm{m}$

B. In a general lattice the center of curvature for the calculation of the electric field continuously changes and has to be re-evaluated every time.

Figure 3. A.General lattice, B.geometry for centers

3 dimensions are

$$
\begin{cases}x:=x+\wp_{x} /\left(U_{o} \gamma\right) c d t & \rightarrow \wp_{x}:=\wp_{x}-e E_{x} 2 c d t \\ y:=y+\wp_{y} /\left(U_{o} \gamma\right) c d t & \rightarrow \wp_{y}:=\wp_{y}-e E_{y} 2 c d t \\ z:=z+\wp_{z} /\left(U_{o} \gamma\right) c d t & \rightarrow \wp_{z}:=\wp_{z}-e E_{z} 2 c d t\end{cases}
$$

A 3-D orbit for a particle injected at some angle (radial $(\hat{x})$ and vertical $(\hat{y})$ betatron oscillations) is shown in Figure 4A

Add an RF cavity. Figure 4B shows an example of RF induced energy oscillations in the lattice. 


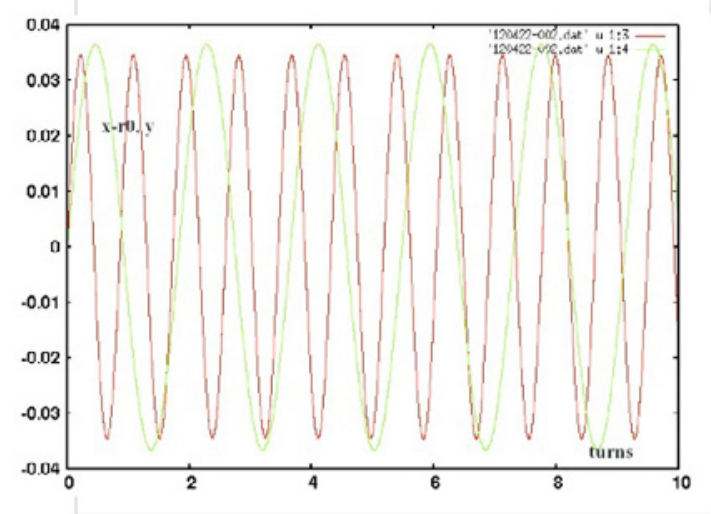

A. $x$ and $y$ betatron oscillations vs. turn

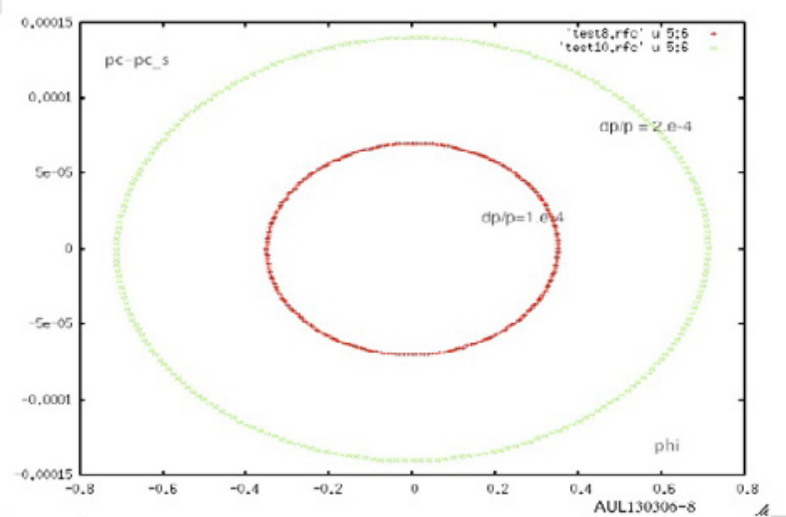

B. RF bucket: Phase space of $\Delta \times \mathrm{pc}$ for two particles, with $\mathrm{dp} / \mathrm{p}=1 . \mathrm{e}-4$ and $2 . \mathrm{e}-4$, $\mathrm{V}=1000$ Volt and $\mathrm{h}=24$.

Number of turns for a complete oscillations 335 corresponding synchrotron frequency $v_{s}=0.002985$ oscillations per turn

Figure 4. A. betatron oscillations, B. RF bucket

\section{Leapfrog magnetic bends}

For benchmarking purposes and test the generality of the algorithm, Leapfrog can be used also to integrate the Lorentz equation in a magnetic lattice. Figures 5. and 6 refer to an example of magnetic bend, in the simple case of a uniform magnetic field parallel to $\hat{y}$, of value i.e. $B \equiv B_{y}$, that should produce an helical trajectory.

It is (see the ménagerie.) $: e B=\frac{\wp}{r_{0} c}$. The momentum kick equations are in $(\mathbf{B})$

$$
\begin{cases}\frac{d p_{x}}{d t}=e B v_{z} & \rightarrow \wp_{x}=\wp \frac{v_{z}}{r} \delta t \\ \frac{d p_{z}}{d t}=-e B v_{x} & \rightarrow \wp_{z}=\wp\left(1-\frac{v_{x}}{r} \delta t\right)\end{cases}
$$

The components of the velocity in $(\mathbf{B})$ are

$$
v_{x}=-v \sin \theta, \quad v_{z}=v \cos \theta .
$$

Comparing (17) with Eq.(8) of the electric case, note the anti-symmetric role of sine and cosine, because the electric bend field is radial, while the velocity is tangential. At the very beginning of tracking, at $\mathbf{B}$, after the drift, it is

$$
v_{x}=0, \quad v_{z}=v
$$

so, according to (16) only $p_{x}$ would receive a kick, which is paradoxical -but a toll one pays using kick formalism- because a magnetic force cannot change the total momentum. After the kick we may therefore write

$$
\wp_{x}=\wp \frac{v}{r_{0}} \delta t, \quad \wp_{z}=\wp \sqrt{1-\left(\wp_{x}^{2} / \wp^{2}\right)}
$$


For a magnetic kick the 1.st leapfrog condition is satisfied for free by default. The 2.nd leapfrog condition for the angle that should lead to a relation between $d t$, the time step for a drift, and $\delta t$, the time step for a magnetic bend.

From Figure 4A, we see that at the beginning the polygonal condition for the kick bend angle requires:

$$
\frac{\wp_{x}}{\wp_{z}}=\tan 2 \theta=\frac{2 \tan \theta}{1-\tan ^{2} \theta}, \text { and }: \tan \theta=\frac{v d t}{r_{0}}=\frac{\ell}{r_{0}},
$$

with $\ell$ the drift length. For subsequent points and in general, the figure shows that Eq.(13) should be replaced by

$$
\frac{\wp_{r}}{\wp_{\theta}}=\frac{2 \tan \theta}{1-\tan ^{2} \theta} .
$$

where $\wp_{r}$ and $\wp_{\theta}$ are the radial and tangential component of the momentum after the kick, respectively

$$
\wp_{r}=\wp_{x} \cos \theta+\wp_{z} \sin \theta, \wp_{\theta}=-\wp_{x} \sin \theta+\wp_{z} \cos \theta
$$

With some algebra, the relation between $\delta t$ and $d t$ that sets the angle condition for a magnetic bend is

$$
\delta t=\frac{2 d t}{1+\left(\ell / r_{0}\right)^{2}}
$$

very close to what was found for the electric bend, Eq.(12).

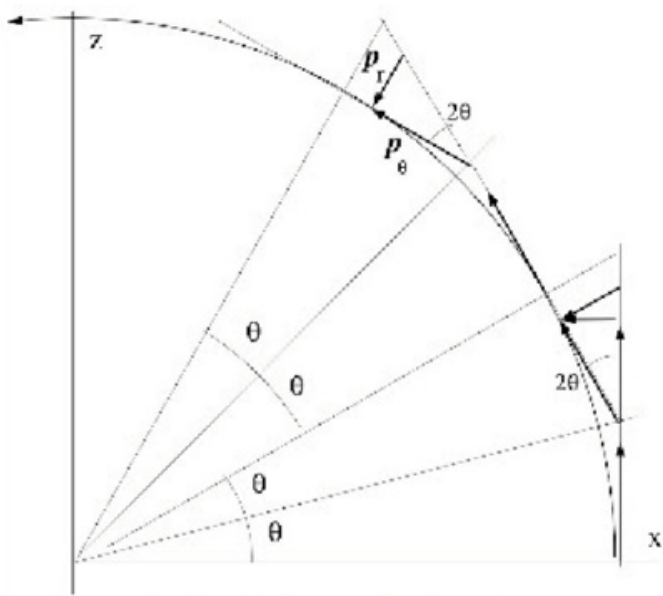

A. Magnetic for condition Leapfrog-2

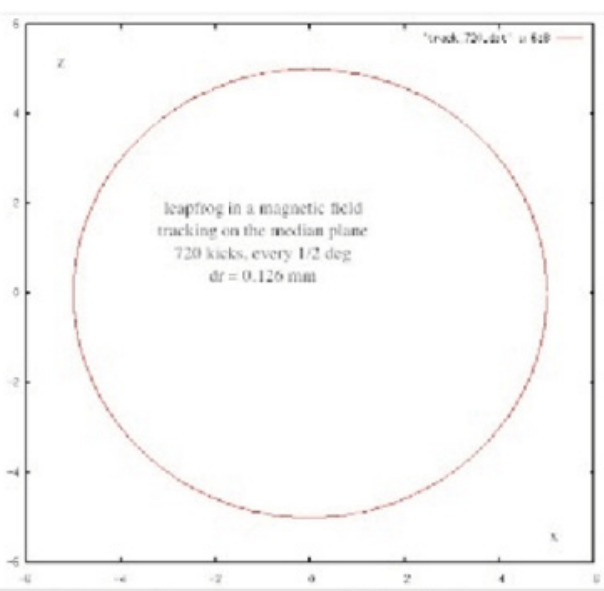

B. Magnetic: trajectory on the $\mathrm{Sx}-\mathrm{z} S$ plane

Figure 5. A.Magnetic-LF2, B.Magnetic trajectory

For the magnetic case it is immediate to see that, directly using Eqs.(16), the 1.st leapfrog condition, i.e the conservation of the momentum in a kick is NOT satisfied, differently than for an electric kick, Eq.(12). A solution is found by combining the two 1.st order ODE's equations into two 2.nd order equations.

Let us re-write Eqs.(16) in the more compact form

$$
\frac{d p_{x}}{d t}=-\omega p_{z}, \frac{d p_{z}}{d t}=\omega p_{x}, \text { with } \omega=\frac{v}{r_{0}} .
$$




\section{ICNFP 2013}

Differentiate both, obtain two formally identical 2.nd order ODE's

$$
\frac{d^{2} p_{x}}{d t^{2}}+\omega^{2} p_{x}, \frac{d^{2} p_{z}}{d t^{2}}+\omega^{2} p_{z}
$$

with integral

$$
p_{x, z}=A_{x, z} \sin (\omega \delta t)+B_{x, z} \cos (\omega \delta t),
$$

or

$$
\left\{\begin{array}{l}
p_{x, n+1}:=p_{x, n} \cos (\omega \delta t)-p_{z, n} \sin (\omega \delta t) \\
p_{z, n+1}:=p_{x, n} \sin (\omega \delta t)+p_{z, n} \cos (\omega \delta t)
\end{array} .\right.
$$

it is immediate to verify that

$$
p_{x, n+1}^{2}+p_{z, n+1}^{2}=p_{x, n}^{2}+p_{z, n}^{2}=p^{2} .
$$

Condition Leapfrog2 is geometrically illustrated by Figure 5A. Figure 5B shows the leapfrog trajectory in the $x-z$ plane for a helix. Parameters, averages and values of various quantities for this track example are given in the following table. The helix created in this magnetic case is shown in Figure

6. Parameters for the magnetic case are:

\begin{tabular}{|ll|}
\hline nsub & $=720: 0.5 \mathrm{deg}$ per bend \\
$r_{o}[\mathrm{~m}]$ & $=5$ \\
$\wp_{0}=p c_{0}[\mathrm{GeV}]$ & $=0.70074037$ \\
$<p c>$ & $=0.70074037 \pm 7.5247175510^{-8}$ \\
$B\left[\right.$ V.s $\left./ m^{2}\right]$ & $=0.46748365$ \\
$<$ angle $>$ & $=5.0626860710^{-5}$ \\
$d r[\mathrm{~mm}]$ & $=0.12692779$ \\
\hline
\end{tabular}

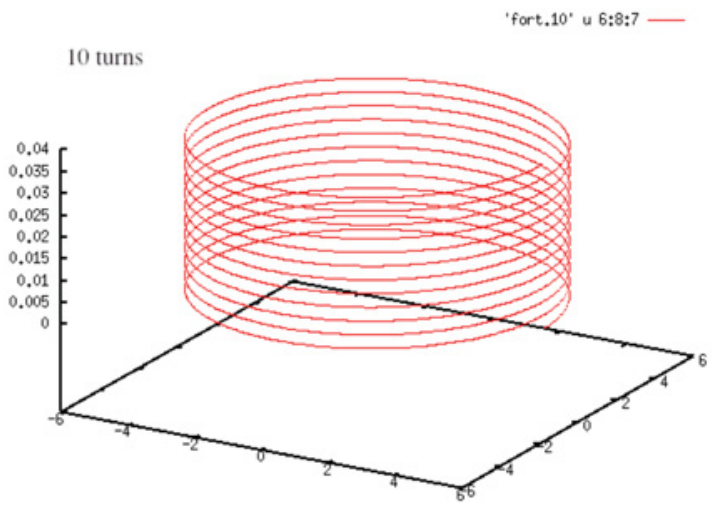

130719_track_2

Figure 6. Magnetic example: 10-turn Helix created with Leapfrog 


\section{Briefly on Spin Dynamics: EDM}

Spin Dynamic is part of the present Leapfrog implementation, following the SPINK algorithms[8]. Spin kicks, applied at each Bend and Quad, follow the leapfrog pattern of the orbit.

At the magic energy it is $\mathbf{F}=\mathbf{0}$ and the spin remains frozen If the proton has an EDM, the spin will not be completely frozen: in the rest frame of the particle, the electric field will appear as a magnetic field $\mathbf{B}^{\prime} \perp$ to $\mathbf{E}$ and another small term is added to $\mathbf{f}$ in Eq.(2)

$$
\mathbf{B}^{\prime}=-\gamma \tilde{\beta} \times \mathbf{E} . \mathbf{f}:=\mathbf{f}+\eta \mathbf{B}^{\prime} \times \mathbf{v} .
$$

The spin will make a precession around this magnetic field and a spin vertical component will appear, that can be measured. For a magic proton this is the only non vanishing additional spin component. Figure.7 shows the frozen spin in accelerator coordinates.

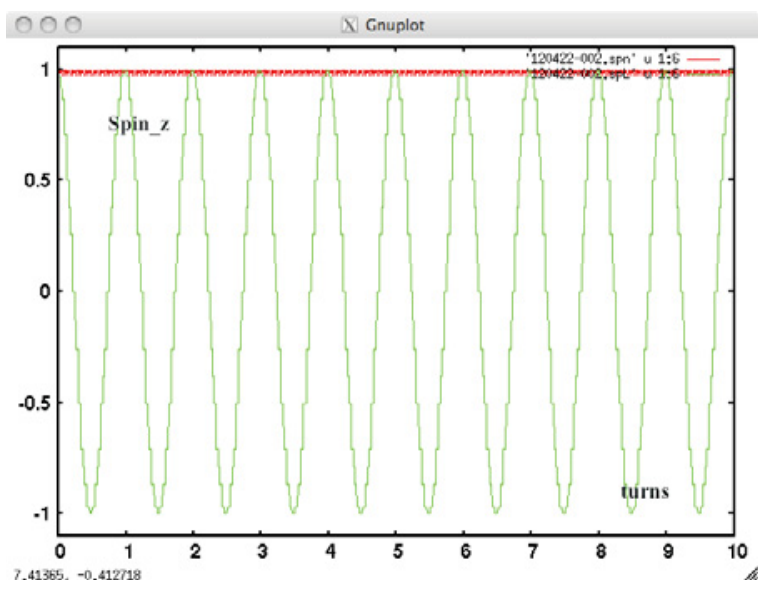

Figure 7. Longit. component of the frozen spin: red line in accelerator coordinates, green line, in laboratory coordinates. The red line shows little wiggles because the responsible proton is on purpose not perfectly magic and there are energy oscillations.

\section{References}

[1] J.B.Delambre, Tables du Soleil (1792)

[2] L.Verlet, Phys.Rev. 159,98-103 (1960)

[3] R.D.Ruth, IEEE Trans. on Nuclear Sciences, NS-3 (1983)

[4] L.Schachinger and R.Talman Particle Accelerators, 22 (1987)

[5] J.M.Sanz-Serna, Acta Numerica, QA297-A21,243-286 (1992)

[6] W.H.Press et Al, Numerical Recipes (Cambridge Uni. Press,1992) p.833

[7] https://www.cern.ch/mad

[8] A.U.Luccio, Proc.Spin08 Charlottesville, AIP Conf.Proc VA 6-11,1149 (2009) 